\title{
Effect of Immediate or Delayed Light Activation on Curing Kinetics and Shrinkage Stress of Dual-Cure Resin Cements
}

\author{
A Faria-e-Silva $\bullet$ L Boaro $\bullet$ R Braga \\ E Piva $\bullet$ V Arias $\bullet$ L Martins
}

\section{Clinical Relevance}

Under clinical conditions, delayed light activation of dual-cured resin cements may be advantageous in order to reduce shrinkage stress.

*Andre Faria-e-Silva, DDS, DMD, PhD, Department of Dentistry, Federal University of Sergipe, Aracaju, SE, Brazil.

Leticia Boaro, DDS, DMD, Department of Dental Materials, School of Dentistry, University of São Paulo, São Paulo, SP, Brazil.

Roberto Braga, DDS, DMD, PhD, Department of Dental Materials, School of Dentistry, University of São Paulo, São Paulo, SP, Brazil.

Evandro Piva, DDS, DMD, PhD, Department of Restorative Dentistry, School of Dentistry, Federal University of Pelotas, Pelotas, RS, Brazil.

Vanessa Arias, DDS, DMD, PhD, Department of Restorative Dentistry, São Leopoldo Mandic School of Dentistry, Campinas, SP, Brazil.

Luis Martins, DDS, DMD, PhD, Department of Restorative Dentistry, State University of Campinas, Piracicaba, Brazil.

*Corresponding author: Department of Dentistry, Rua Cláudio Batista S/N, Aracaju, SE, Brazil, 49060-100, email: faria_silva@ufs.br

DOI: $10.2341 / 10-153-\mathrm{L}$

\section{SUMMARY}

Objectives: This study evaluated the effect of light activation (absence, immediate, or delayed) on conversion kinetics and polymerization stress of three commercial dual-cured resin cements (Enforce, RelyX ARC, and Panavia F). Methods: Degree of conversion (DC) was monitored for $\mathbf{3 0}$ minutes using real-time nearFourier transform infrared spectroscopy. The cement was mixed, placed on the spectrometer sample holder, and light activated either immediately or after five minutes (delayed light activation). When no light activation was performed, the materials were protected from light exposure (control). DC was evaluated at five and 30 minutes postmixture. Maximum rates of polymerization $\left(R p_{\text {max }}\right.$ ) were obtained from the first derivative of the $D C$ vs time curve. Polymerization stress was monitored for 30 minutes in 1-mm-thick specimens inserted between two cylinders attached to a uni- 
versal testing machine. Data were submitted to analysis of variance/Tukey tests $(\alpha=0.05)$. Results: Immediate light activation promoted the highest DC at five minutes. At 30 minutes, only RelyX ARC did not present a significant difference in DC between activation modes. Enforce and Panavia $F$ presented higher $R p_{\max }$ for immediate and delayed light-activation, respectively. RelyX ARC showed similar $\mathbf{R p}_{\max }$ for all activation modes. The absence of light activation resulted in the lowest stress followed by delayed light activation, while immediate light activation led to the highest values. RelyX ARC showed higher stress than Enforce, while the stress of Panavia F was similar to that of the others. Conclusion: Delayed light activation reduced the polymerization stress of the resin cements tested without jeopardizing DC.

\section{INTRODUCTION}

During post cementation, the exposed cervical margin is readily accessible to the curing light. However, a significant reduction in irradiance occurs as a result of the light scattering within the resin cement itself and shadowing produced by both tooth structure and the post. ${ }^{1}$ In this situation, dual-cure resin cements are indicated, as they provide optimal polymerization, either in the presence or the absence of the curing light. Nevertheless, some dual-cured resin cements are dependent primarily on light activation, and therefore a somewhat lower degree of conversion is expected when exposed to low irradiance or when light from the curing unit is not available at all. ${ }^{2-6}$

Other studies found similar properties between dual-cure resin cements tested after light activation or in the self-cure mode. ${ }^{7,8}$ It has been hypothesized that light activation may negatively affect the selfcuring mechanism. The rationale is that the rapid formation of a cross-linked polymer on exposure to light would lead to entrapment of the reactive species, including activators and initiators needed for the selfcure reaction. ${ }^{3,9-12}$ Thus, a delay period between cement mixing and light activation would increase total free-radical concentration, which would lead to a higher overall degree of conversion, ultimately improving the cement's mechanical properties. ${ }^{3,13}$

Unfortunately, a higher degree of conversion is also related to an increase in polymerization shrinkage stress. ${ }^{14-16}$ If stress exceeds the bond strength between the dental substrate and the adhesive system, a contraction gap will be formed, ${ }^{17-20}$ jeopardizing the longevity of the restoration. It has been demonstrated that lower polymerization rate $^{21,22}$ and lower elastic modulus ${ }^{22-25}$ may help reduce polymerization stress. Therefore, it is important to determine the effect of delayed light activation on polymerization reaction rate and stress development in comparison with immediate light activation of these types of products.

The purpose of this study was to investigate the effect of immediate and delayed light activation on polymerization kinetics and shrinkage stress of three commercially available, dual-cured resin cements. For control purposes, the cements were also tested in the absence of light. It was hypothesized that delaying the light activation of dual cured resin cements 1) does not compromise the degree of conversion, 2) reduces the maximum rate of polymerization, and 3) reduces polymerization stress.

\section{METHODS AND MATERIALS}

\section{Degree of Conversion and Maximum Polymerization Rate ( $\mathbf{R P}_{\text {max }}$ )}

Names and composition of the dual-cured resin cements tested are listed in Table 1. Degree of conversion was assessed using real-time near-Fourier transform infrared spectroscopy (Vertex 70, Bruker Optik GmbH, Ettlingen Germany). The spectrometer used an extended $\mathrm{KBr}$ beam splitter (Bruker Optik) and a InGaAs detector (Bruker Optik). Spectra (two per second) were obtained between $4000 \mathrm{~cm}^{-1}$ and $9840 \mathrm{~cm}^{-1}$, with a $4-\mathrm{cm}^{-1}$ resolution. For all resin cements, equal volumes of base and catalyst were mixed for 15 seconds and inserted into a 0.8-mm-thick circular silicone mold (with a 3.5-mm radius) placed on a glass slide. A second glass slide was placed on top of the mold, and the assembly was stabilized with clamps before positioned on the spectrometer sample holder.

The following polymerization scenarios were tested for all resin cements:

- Immediate light activation: The resin cements were immediately light activated, according to the manufacturers' recommended times: 20s (Panavia F), 30s (Enforce), and 40s (RelyX ARC).

- Delayed light activation: The luting agents were light activated 5 minutes after beginning the mixing procedure for the same time durations described above. This delay period was chosen on the basis that it allowed the resin cements to achieve more than $50 \%$ of their maximum degree of conversion in the self-cure mode (pilot study). 
Table 1: Materials Used

\begin{tabular}{|c|c|c|c|c|}
\hline \multirow[t]{2}{*}{ Enforce } & Dentsply & $\begin{array}{l}\text { Base: Bis-GMA, TEGDMA, CQ, EDAB, BHT } \\
\text { and DHEPT }\end{array}$ & Translucent & 66 \\
\hline & (Petrópolis, RJ, Brazil) & $\begin{array}{l}\text { Catalyst: Bis-GMA, BHT, EDAB, TEGDMA } \\
\text { and BPO }\end{array}$ & & \\
\hline \multirow[t]{2}{*}{ RelyX ARC } & 3M ESPE & $\begin{array}{l}\text { Paste A: Bis-GMA, TEGDMA, dimethacrylate } \\
\text { polymer, } C Q \text {, amine }\end{array}$ & Transparent (A1) & 67.5 \\
\hline & (St. Paul, MN, USA) & $\begin{array}{l}\text { Paste B: Bis-GMA, TEGDMA, dimethacrylate } \\
\text { polymer, BPO }\end{array}$ & & \\
\hline \multirow[t]{2}{*}{ ED Primer } & Kuraray Co. & $\begin{array}{l}\text { Primer A: HEMA, 10-MDP, NM-aminosalicilic } \\
\text { acid, DHEPT, water }\end{array}$ & & - \\
\hline & & $\begin{array}{l}\text { Primer B: NM-aminosalicilic acid, T- } \\
\text { isopropylic benzenic sodium sulfinate, } \\
\text { DHEPT, water }\end{array}$ & & \\
\hline \multicolumn{5}{|c|}{$\begin{array}{l}\text { Abbreviations: Bis-GMA, bisphenol-A glycidyl dimethacrylate; TEGDMA, triethylene glycol dimethacrylate; CQ, camphorquinone; EDAB, ethyl 4-dimethylamine b; BHT, } \\
\text { butylhydroxytoluene; DHEPT, N,N-di-(2-hydroxyethyl)-4-toluidine; BPO, benzoylperoxide; 10-MDP, 10-methacryloyloxydecyl dihydrogenphosphate; HEMA, } \\
\text { hydroxyethyl methacrylate. } \\
\text { a Information provided by the manufacturer. }\end{array}$} \\
\hline
\end{tabular}

- Chemical activation: No light activation was performed, and polymerization relied on selfactivation only.

Panavia F was mixed with ED Primer before the evaluation, as the manufacturer states that the primer is essential for proper cement polymerization. ${ }^{15}$ For this material, one drop of each of the primer liquids A and B was mixed for 10 seconds and gently air-dried for 5 seconds to evaporate the solvent. Thereafter, $0.1 \mu \mathrm{L}(1.4 \mathrm{mg})$ of this solution was dispensed onto a glass slab using a micropipette (model NPX2; Nichipet EX, Santa Clara, CA, USA) and mixed with the base and catalyst pastes $(27 \mathrm{mg}$ of each paste) of the dual-cure cement for 15 seconds. The volume of ED Primer added to the cement was based in a previous study. ${ }^{26}$

All analyses were performed under controlled temperature $\left(25 \pm 1^{\circ} \mathrm{C}\right)$ and humidity $(60 \pm 5 \%)$ conditions. The output of the light-curing unit (Optilux 501; Demetron Kerr, Orange, CA) was periodically checked using a handheld radiometer (Model 100; Demetron Kerr) and was determined to be near 800 $\mathrm{mW} / \mathrm{cm}^{2}$. The tip of the light guide was placed at a 45-degree angle with the cement specimen, $1 \mathrm{~mm}$ distant from the glass slide, in order to not interfere with the infrared beam. For the immediate lightactivated groups, the curing light was triggered after the first two spectra were collected, as those spectra were used as the unpolymerized reference. The materials were shielded from ambient light for the self-cure and delayed light-activation modes during all the experiment and in the first five minutes, respectively. Shielding was performed by keeping the material inside the closed sample compartment.

Spectra were obtained for 30 minutes. The area under the peak located at $6165 \mathrm{~cm}^{-1}$ corresponding to the first overtone of the $\mathrm{C}-\mathrm{H}$ stretch $^{27}$ of the vinyl group was integrated with the use of software (Opus v.6; Bruker Optik). Degree of conversion was calculated by dividing the area of one spectrum 
obtained at a given time by the average area of the first two spectra (ie, corresponding to the unpolymerized cement). Three replicates were obtained for each experimental condition. Degree of conversion values corresponding to five minutes and 30 minutes were recorded. The maximum polymerization rate $\left(\mathrm{RP}_{\max }\right)$ was calculated as the first derivative of the conversion vs time curve. ${ }^{27}$

\section{Polymerization Stress}

Polymerization stress was measured using a universal testing machine (Instron 5565; Instron, Canton, MA, USA). The test was performed using clear poly(methyl methacrylate) (PMMA) ${ }^{28}$ cylinders (6 $\mathrm{mm}$ in diameter and 13 or $28 \mathrm{~mm}$ high) as bonding substrates. One flat end of the short cylinders was polished using 600 - to 1200 -grit $\mathrm{SiC}$ abrasive paper in order to allow the highest possible light transmission during light activation. The other end of the short cylinders and both ends for the long ones were airborne particle abraded with $100 \mu \mathrm{m}$ aluminum oxide. Afterward, methyl methacrylate monomer (Jet Acrílico Autopolimerizante; Artigos Odontológicos Clássico, São Paulo, Brazil) was applied to improve the wettability of the PMMA surface. The surfaces were then covered with a layer of unfilled bonding resin (Scotchbond Multi-purpose Plus; 3M ESPE, St. Paul, MN, USA), which was then light cured for 20 seconds.

The 28-mm cylinder was clamped to the upper chuck, while the $13-\mathrm{mm}$ cylinder was attached to a stainless-steel fixture clamped to the lower chuck. The resin cements were mixed in the same manner as described for polymerization kinetics evaluation and inserted between the PMMA cylinders, with the distance set to $1 \mathrm{~mm}$. An extensometer (model 2630101; Instron) was attached to the cylinders in order to keep specimen height constant during the test. Any approximation between the fixation points of the extensometer caused by resin cement shrinkage was immediately compensated for by controlled movement of the crosshead in the opposite direction, with accuracy of $0.1 \mu \mathrm{m}$.

Resin cements used in the dual-cured mode were light activated with the same curing unit and for the same exposures described above. However, because light was shone through the clear PMMA rod, the irradiance effectively reaching the resin cement was $570 \mathrm{~mW} / \mathrm{cm}^{2}$ (as measured by a dental radiometer). The light guide was placed in contact with the polished end of the lower cylinder. Software (Bluehill 2 ; Instron) was used to monitor stress development. The test was conducted at room temperature $\left(25 \pm 1^{\circ} \mathrm{C}\right)$, and data were recorded for 30 minutes from the time of resin cement placement. The value recorded by the load cell corresponded to the force necessary to keep specimen height constant, in opposition to the force exerted by composite shrinkage. The maximum nominal polymerization stress was calculated by dividing the maximum force value by the cross-sectional area of the cylinder. Five specimens were tested for each experimental condition. Stress vs time data were plotted, and Hill's 3 parameter nonlinear regressions were used for curve fitting (Sigmaplot 10.0; Systat Software Inc, San Jose, CA, USA).

\section{Statistical Analysis}

Degree of conversion, $\mathrm{RP}_{\max }$, and polymerization stress data were each subjected to two-way analysis of variance (ANOVA; cement and curing method as main factors) using statistical software Sigmastat 3.5 (Systat Software Inc, San Jose, CA, USA). Multiple pairwise comparisons were performed using Tukey post hoc test. In all cases, a preset global significance level of 5\% was adopted.

\section{RESULTS}

\section{Degree of Conversion and Maximum Polymerization Rate}

Degree of conversion at five minutes (Table 2) showed a significant effect for the factor "activation mode" ( $p=0.153$ ) and for the interaction between the factors ( $p=0.002$ ) but not for the factor "resin cement" $(p=0.153)$. For Enforce and Panavia F, immediate light activation resulted in statistically higher conversion values compared to delayed light activation and self-curing mode. RelyX ARC displayed

\begin{tabular}{|lccc|}
\hline Table 2: & $\begin{array}{l}\text { Means }( \pm S D) \text { of Percent Degree of Conversion } \\
\text { at Five Minutes }{ }^{a}\end{array}$ \\
\cline { 2 - 4 } & \multicolumn{3}{c|}{ Resin Cements } \\
\cline { 2 - 4 } Activation Mode & Enforce & RelyX ARC & Panavia F \\
\hline $\begin{array}{l}\text { Immediate light } \\
\text { activation }\end{array}$ & $72.8(3.4) \mathrm{Aab}$ & $59.7(6.3) \mathrm{Ab}$ & $82.4(4.1) \mathrm{Aa}$ \\
\hline $\begin{array}{l}\text { Delayed light } \\
\text { activation }\end{array}$ & $37.6(10.7) \mathrm{Ba}$ & $39.7(13.1) \mathrm{Ba}$ & $28.0(10.6) \mathrm{Ba}$ \\
\hline $\begin{array}{l}\text { Self-cure } \\
\text { 47.6 (3.5) Ba }\end{array}$ & $44.0(2.3) \mathrm{Ba}$ & 26.5 (4.8) Bb \\
\hline $\begin{array}{l}\text { a Means followed by the same letters (uppercase, column; lowercase, row) } \\
\text { are not statistically different (p>0.05). }\end{array}$ \\
\hline
\end{tabular}


similar conversion at five minutes among the three activation modes. However, cements ranked differently in each curing mode. With immediate light activation, DC of Panavia F reached the highest conversion, not significantly different from Enforce, while RelyX ARC was similar only to the latter. When light activation was delayed or in self-cure mode, conversion values for all three cements were not significantly different.

Conversion values obtained at 30 minutes are shown in Table 3. The factors "resin cement" $(p<0.001)$ and "activation mode" $(p<0.001)$ were significant, as was their interaction $(p=0.003)$. For immediate light activation, Panavia $\mathrm{F}$ showed the highest values of DC, while RelyX ARC reached the lowest values. Conversion values for Enforce and RelyX ARC were not significantly different for delayed light activation and self-cure modes. Panavia $\mathrm{F}$ reached highest conversion when tested in delayed light-activation mode and similar values to other cements in self-cure mode. Comparison among cements revealed that Panavia $\mathrm{F}$ reached the highest conversion in both light-cure modes, while conversion values for Enforce and RelyX ARC were not significantly different. Conversion values 30 minutes postmix among the three cements using the self-cure mode were not significantly different.

For the maximum rate of polymerization $\left(\mathrm{RP}_{\max }\right)$, the statistical analysis also showed significant effects for the factors "resin cement" $(p<0.001)$ and "activation mode" ( $p=0.002)$ and for the interaction between the factors $(p<0.001$; Table 4$)$. For Enforce, reaction rate was significantly higher for specimens immediately light activated, while delayed lightactivation and self-curing values were not significantly different. The $\mathrm{Rp}_{\max }$ for RelyX ARC was not

\begin{tabular}{|c|c|c|c|}
\hline \multirow[b]{2}{*}{ Activation Mode } & \multicolumn{3}{|c|}{ Resin Cements } \\
\hline & Enforce & RelyX ARC & Panavia F \\
\hline $\begin{array}{l}\text { Immediate light } \\
\text { activation }\end{array}$ & 74.8 (2.2) Ab & 65.4 (6.7) Ac & $89.0(2.4) \mathrm{Aa}$ \\
\hline $\begin{array}{l}\text { Delayed light } \\
\text { activation }\end{array}$ & $72.5(0.4) \mathrm{ABb}$ & 72.2 (4.9) Ab & $93.4(3.0) \mathrm{Aa}$ \\
\hline Self-cure & $67.1(1.0) \mathrm{Ba}$ & 66.4 (3.9) $\mathrm{Aa}$ & $73.4(3.5) \mathrm{Ba}$ \\
\hline
\end{tabular}

\begin{tabular}{|c|c|c|c|}
\hline \multirow[b]{2}{*}{ Activation Mode } & \multicolumn{3}{|c|}{ Resin Cements } \\
\hline & Enforce & RelyX ARC & Panavia F \\
\hline $\begin{array}{l}\text { Immediate light } \\
\text { activation }\end{array}$ & 9.7 (1.7) Ab & 10.5 (1.6) Ab & $21.0(1.2) \mathrm{Ba}$ \\
\hline $\begin{array}{l}\text { Delayed light } \\
\text { activation }\end{array}$ & $1.0(0.1) \mathrm{Bc}$ & $9.2(4.3) \mathrm{Ab}$ & 29.1 (3.1) Aa \\
\hline Self-cure & $0.8(0.2) \mathrm{Bc}$ & $7.8(1.5) A b$ & 21.0 (1.7) Ba \\
\hline
\end{tabular}

significantly different among all activation modes. Panavia $\mathrm{F}$ showed higher $\mathrm{RP}_{\max }$ for the delayed activation mode than using either of the other two modes. Comparison of $\mathrm{RP}_{\max }$ among cements revealed that Panavia $F$ had the highest value regardless of the curing method, followed by RelyX $\mathrm{ARC}$ and Enforce. No significant difference in $\mathrm{RP}_{\max }$ was observed between the latter two cements when using immediate light activation.

Representative rate of polymerization profiles for the different polymerization scenarios are shown in Figures 1 and 2 . For all resin cements, the peak of $\mathrm{RP}_{\text {max }}$ occurred within the first few seconds of the reaction when light activation was applied immediately. The peak in the polymerization rate curve was displaced to the right when the resin cements were only chemically activated. The delayed light activa-

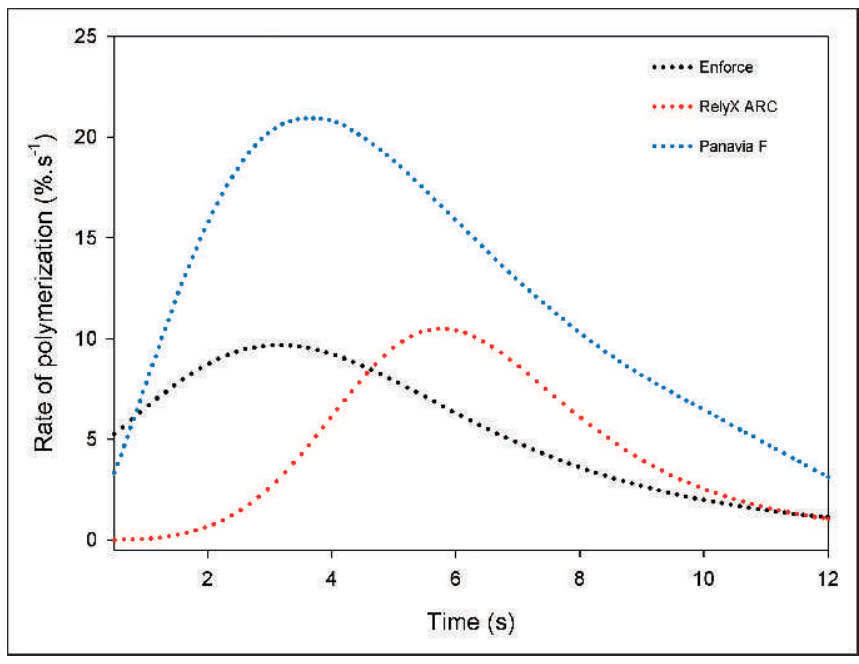

Figure 1. Representative polymerization rate profiles for the immediate light-activation mode. 


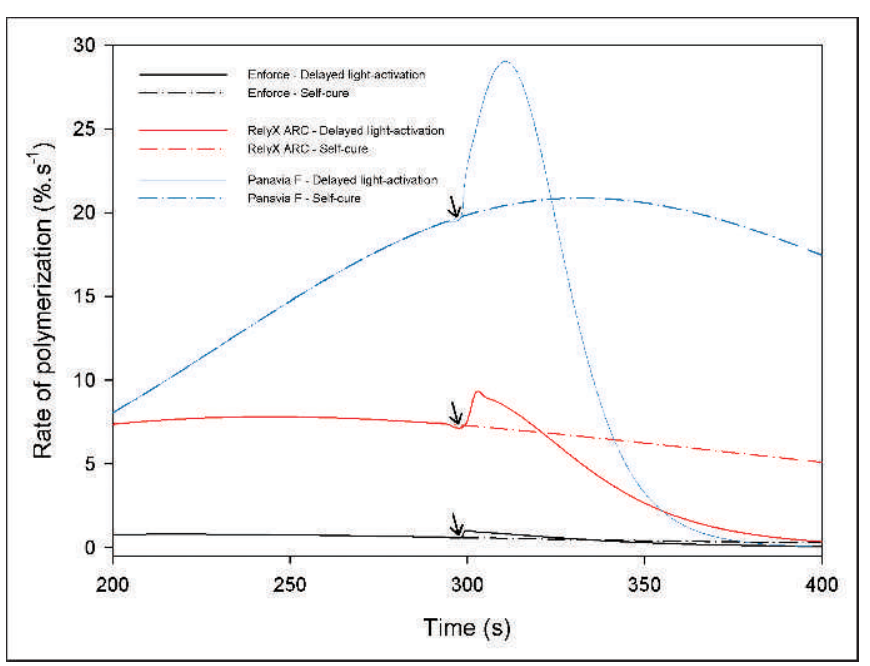

Figure 2. Representative polymerization rate profiles for the selfcured and delayed light-activated groups. Arrows indicate the moment when the light activation was performed.

tion generated a second peak during the autodeceleration of polymerization reactions for RelyX ARC and Enforce, while this second peak was observed during autoacceleration for Panavia F.

\section{Polymerization Stress}

ANOVA showed significant effect for the main factors on maximum polymerization stress values (resin cement: $p=0.024$; activation mode: $p<0.001$ ) but not for their interaction $(p=0.46)$. Pairwise means comparisons are shown in Table 5. RelyX ARC presented higher maximum polymerization stress than Enforce. Panavia F showed intermediate values without significant differences from the other resin cements. For all three resin cements, the highest stress values were obtained with immediate light activation. The use of delayed light activation resulted in intermediate stress values, while the lowest values were detected when no light activation was performed. A $19 \%$ reduction in stress was seen using the delayed mode compared to immediate light activation, while when used as self-curing materials, nominal stress values were four times lower than with immediate light activation.

The stress development profiles are illustrated in Figure 3. The coefficient of determination was greater than 0.9 for all curves. For the immediate light-activation mode, a fast increase in stress was observed in the first seconds. After this period, the slope of the curve decreased, reaching a plateau around 200 seconds for all cements. A gradual increase in stress was observed in the absence of light activation for all cements, reaching a plateau later (around 1200 seconds for Panavia F and RelyX ARC and 800 seconds for Enforce). A rapid increase in stress was observed at 300 seconds for the delayed light-activation mode, reaching a plateau around 900 seconds for all cements.

\section{DISCUSSION}

The results indicated that, among the three cements tested, only RelyX ARC reached similar conversion levels for all activation modes. Delayed light activation did not affect the degree of conversion after 30 minutes for any of the materials. Thus, the first hypothesis was confirmed. As expected, conversion values measured at five minutes were higher for the immediate light-activation and statistically similar for the delayed light-activation and self-cure groups. After 30 minutes, the statistically similar conversions for both immediate and delayed light-activated cements suggest that the possible increased mobility of the reaction media, caused by postponing light

Table 5: Means $( \pm S D)$ of Maximum Polymerization Stress $(M P a)^{a}$

\begin{tabular}{|llll|}
\hline \multirow{2}{*}{ Activation Mode } & \multicolumn{3}{c|}{ Resin Cement } \\
\cline { 2 - 4 } & Enforce & RelyX ARC & Panavia F \\
\hline Immediate light activation & $4.7(0.1)$ & $4.9(0.2)$ & $4.9(0.3)$ \\
\hline Delayed light activation & $3.8(0.2)$ & $4.2(0.6)$ & $3.7(0.3)$ \\
\hline Self-cure & $0.9(0.2)$ & $1.4(0.4)$ & $1.2(0.2)$ \\
\hline Pooled average & $3.1(1.7) \mathrm{B}$ & $3.5(1.6) \mathrm{A}$ & $3.3(1.6) \mathrm{AB}$ \\
\hline a For the pooled averages, means followed by the same letters are not statistically different (p>0.05). & \\
\hline
\end{tabular}




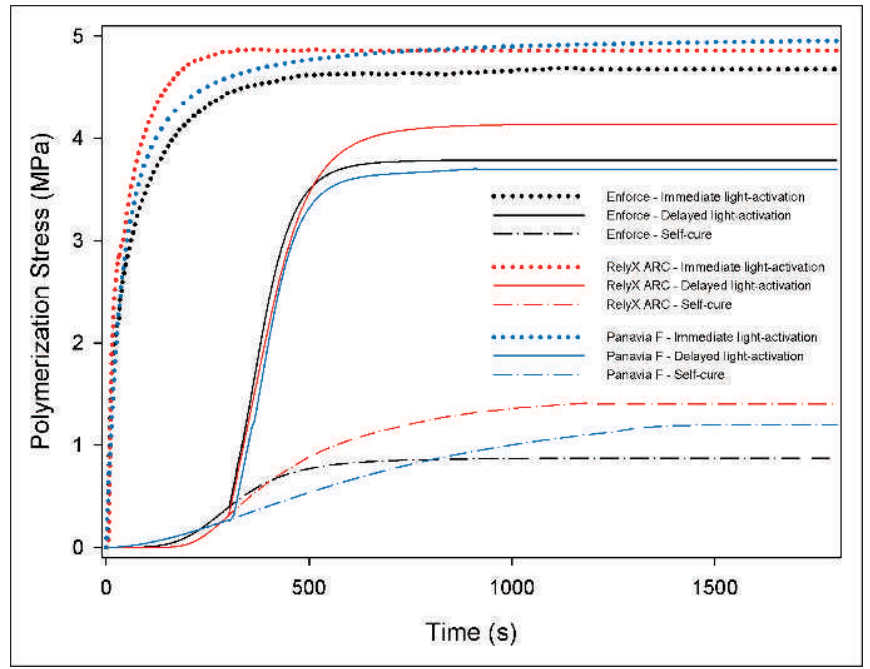

Figure 3. Representative polymerization stress development profiles.

activation, did not contribute to the extent of cure. Others also did not find differences between delayed and immediate light activation of one experimental dual-cured resin cement when a high-energy dose was used for light activation, as in the present study. ${ }^{3}$

The delay period before light activation significantly reduced the $\mathrm{RP}_{\text {max }}$ for Enforce to a value statistically similar to the reaction rate of the cements tested in self-cure mode. RelyX ARC showed statistically similar $\mathrm{RP}_{\max }$ in all three curing modes, and Panavia $\mathrm{F}$ showed the highest values for delayed light activation. Thus, the second hypothesis tested was rejected. The increase in $\mathrm{RP}_{\max }$ using the delayed light activation for Panavia $\mathrm{F}$, different from the other cements, can be related to the lowerenergy dose used for light activation (20 seconds), as previously demonstrated. ${ }^{3}$ A low $\mathrm{RP}_{\max }$ indicates a slow polymerization reaction, which may help to reduce polymerization stress. During the polymerization reaction, viscosity and elastic modulus increase ${ }^{9,10}$ Delaying the acquisition of a high elastic modulus allows the polymer chains to rearrange and accommodate the reduction of volume by plastic deformation. ${ }^{15}$ Furthermore, polymerization stress is related mainly to volumetric shrinkage and elastic modulus, both these being closely related to degree of conversion. ${ }^{15}$

Panavia $\mathrm{F}$ reached the highest values of $R \mathrm{P}_{\text {max }}$ and degree of conversion when the cements were light activated. However, polymerization stress of Panavia F was not different from that of the other cements. The inorganic content of resin composites has an important effect on elastic modulus. ${ }^{24}$ High filler content is associated with stiffer polymers and higher polymerization stress development. ${ }^{25}$ Thus, it could be expected that Panavia F would present the highest stress, which did not occur. Monomer content also has an influence on stress. Less rigid monomers develop polymers with lower elastic moduli, which may also affect polymerization stress development. ${ }^{15}$ However, because the exact formulation of the evaluated cements is known, explaining the differences in stress among commercial materials based on their organic and inorganic contents is merely speculative.

Regardless of the differences detected for $\mathrm{RP}_{\mathrm{m}}$ and degree of conversion, the self-cure mode yielded the lowest polymerization stress for all cements. Similarly, delayed light activation showed lower polymerization stress than immediate light-activation. Thus, the third hypothesis was accepted. As Enforce showed similar degrees of conversion between immediate and delayed light activation, differences in polymerization stress can be attributed to a reduced reaction rate. For Panavia F, the lower polymerization stress observed with self-cure mode can be credited to a lower degree of conversion. RelyX ARC showed similar values of $\mathrm{DC}$ and $\mathrm{RP}_{\max }$ for all curing modes.

It is challenging to explain the differences in stress observed between immediate and delayed light activation, as conversion was similar and $\mathrm{RP}_{\max }$ values were higher for the delayed light activation. In addition to $\mathrm{RP}_{\max }$ and conversion, the polymer structure may also affect stress. Polymers with similar conversion may have different cross-link density. ${ }^{11}$ Differences in polymer cross-link density influence elastic modulus, which also affects polymerization stress. ${ }^{22}$ Therefore, it is only possible to speculate that the delayed light activation could have altered the polymer structure in a way that elastic modulus would be lower, contributing to a reduced stress. The high degrees of conversion and $\mathrm{RP}_{\text {max }}$ values found with Panavia $\mathrm{F}$ may be explained by the fact that the cement was mixed with its primer, which made it less viscous, therefore allowing more carbon double-bond conversion prior to vitrification.

Clinically, dual-cure resin cements must be exposed to the curing light whenever possible. Light attenuation in clinical situations may be compensated for, to some extent, by increasing exposure time. Several studies have shown that marginal defects are frequently present in indirect restorations. ${ }^{18-20}$ The results of this study showed strategies, such as delayed light activation, that may help reduce 
polymerization stress and, ultimately, reduce marginal defects. The delay period used in this study was chosen because it is clinically feasible. However, only one delay period was evaluated, and the effect of other time periods should be evaluated. Another limitation of this study was that shrinkage stress was determined using 1-mm-thick specimens, much thicker than cement layers obtained clinically. Furthermore, the $30 \%$ attenuation in light activation through the PMMA rod limits the correlation between the values of polymerization stress and degree of conversion.

\section{CONCLUSION}

Based on the limitations imposed in the present study, the following conclusions may be drawn:

1. Delayed light-activation did not affect the degree of conversion of the resin cements. Only RelyX ARC reached similar conversion in either the presence or the absence of light, while Panavia F presented the highest conversion values for all curing modes.

2. The maximum rate of polymerization was affected by the activation mode, except for RelyX ARC. Enforce presented a higher rate when immediate light activation was performed, while Panavia F showed higher values for the delayed light activation.

3. Polymerization stress was significantly affected by the activation mode for all resin cements. Immediate light activation generated the highest values, and the lowest polymerization stress was obtained when no light activation was performed.

\section{Acknowledgements}

This study was supported by FAPESP grants \# 2007/ 01006-1 and 2007/00998-0.

(Accepted 27 October 2010)

\section{REFERENCES}

1. Chen YC, Ferracane JL \& Prahl AS (2005) A pilot study of a simple photon migration model for predicting depth of cure in dental composite Dental Materials 21(11) 1075-1086.

2. Fariaesilva AL, Arias VG, Soares LE, Martin AA \& Martins LR (2007) Influence of fiber-post translucency on the degree of conversion of a dual-cured resin cement Journal of Endodontics 33(3) 303-305.

3. Moraes RR, Faria-e-Silva AL, Ogliari FA, Correr-Sobrinho L, Demarco FF \& Piva E (2009) Impact of immediate and delayed light activation on self-polymerization of dual-cured dental resin luting agents Acta Biomaterialia 5(6) 2095-2100.
4. Kim YK, Kim SK, Kim KH \& Kwon TY (2009) Degree of conversion of dual-cured resin cement light-cured through three fibre posts within human root canals: An ex vivo study International Endodontic Journal 42(8) 667-674.

5. Spinell T, Schedle A \& Watts DC (2009) Polymerization shrinkage kinetics of dimethacrylate resin-cements Dental Materials 25(8) 1058-1066.

6. Arrais CA, Giannini M \& Rueggeberg FA (2009) Effect of sodium sulfinate salts on the polymerization characteristics of dual-cured resin cement systems exposed to attenuated light-activation Journal of Dentistry 37(3) 219-227.

7. Braga RR, Condon JR \& Ferracane JL (2002) In vitro wear simulation measurements of composite versus resinmodified glass ionomer luting cements for all-ceramic restorations Journal of Esthetic and Restorative Dentistry 2002 ; 14(6) 368-376.

8. Caughman WF, Chan DC \& Rueggeberg FA (2001) Curing potential of dual-polymerizable resin cements in simulated clinical situations Journal of Prosthetic Dentistry 86(1) 101-106.

9. De Jager N, Pallav P \& Feilzer AJ (2004) The apparent increase of the Young's modulus in thin cement layers Dental Materials 20(5) 457-462.

10. De Jager N, Pallav P \& Feilzer AJ (2005) Finite element analysis model to simulate the behavior of luting cements during setting Dental Materials 21(11) 1025-1032.

11. Soh MS \& Yap AU (2004) Influence of curing modes on crosslink density in polymer structures Journal of Dentistry 32(4) 321-326.

12. Elliott JE, Lovell LG \& Bowman CN (2001) Primary cyclization in the polymerization of Bis-GMA and TEGDMA: A modeling approach to understanding the cure of dental resins Dental Materials 17(3) 221-229.

13. Stavridakis MM, Kakaboura AI \& Krejci I (2005) Degree of remaining $\mathrm{C}=\mathrm{C}$ bonds, polymerization shrinkage and stresses of dual-cured core build-up resin composites Operative Dentistry 30(4) 443-452.

14. Gonçalves F, Pfeifer CS, Ferracane JL \& Braga RR (2008) Contraction stress determinants in dimethacrylate composites Journal of Dental Research 87(4) 367-371.

15. Gonçalves F, Pfeifer CC, Stansbury JW, Newman SM \& Braga RR (2010) Influence of matrix composition on polymerization stress development of experimental composites Dental Materials 26(7) 697-703.

16. Braga RR, Ballester RY \& Ferracane JL (2005) Factors involved in the development of polymerization shrinkage stress in resin-composites: A systematic review Dental Materials 21(10) 962-970.

17. Papadogiannis D, Kakaboura A, Palaghias G \& Eliades G (2009) Setting characteristics and cavity adaptation of low-shrinking resin composites Dental Materials 25(12) 1509-1016.

18. Clelland NL, Ramirez A, Katsube N \& Seghi RR (2007) Influence of bond quality on failure load of leucite- and 
lithia disilicate-based ceramics Journal of Prosthetic Dentistry 97(1) 18-24.

19. Guess PC, Strub JR, Steinhart N, Wolkewitz M \& Stappert CF (2009) All-ceramic partial coverage restorations-Midterm results of a 5-year prospective clinical splitmouth study Journal of Dentistry 37(8) 627-637.

20. Peumans M, De Munck J, Van Landuyt K, Poitevin A, Lambrechts P \& Van Meerbeek B (2010) Two-year clinical evaluation of a self-adhesive luting agent for ceramic inlays Journal of Adhesive Dentistry 12(2) 151-161.

21. Braga RR, Ferracane JL \& Condon JR (2002) Polymerization contraction stress in dual-cure cements and its effect on interfacial integrity of bonded inlays Journal of Dentistry 30(7-8) 333-340.

22. Feng L \& Suh BI (2006) The effect of curing modes on polymerization contraction stress of a dual cured composite Journal of Biomedical Materials Research Part B: Applied Biomaterials 76(1) 196-202.

23. Feng L \& Suh BI (2006) A mechanism on why slower polymerization of a dental composite produces lower contraction stress Journal of Biomedical Materials Research Part B: Applied Biomaterials 78(1) 63-69.

24. Tanimoto T, Nishiwaki K, Nemoto K \& Ben G (2004) Effect of filler content on bending properties of dental composites: Numerical simulation with the use of the finite-element method Journal of Biomedical Materials Research Part B: Applied Biomaterials 71(1) 188-195.

25. Gonçalves F, Kawano Y \& Braga RR (2010) Contraction stress related to composite inorganic content Dental Materials 26(7) 704-709.

26. Faria-e-Silva AL, Moraes RR, Ogliari FA, Piva E, Martins LR (2009) Panavia F: The role of ED Primer Journal of Oral Science 51(2) 255-259.

27. Stansbury JW \& Dickens SH (2001) Determination of double bond conversion in dental resins by near infrared spectroscopy Dental Materials 17(1) 71-79.

28. Gonçalves F, Pfeifer CS, Meira JB, Ballester RY, Lima RG \& Braga RR (2008) Polymerization stress of resin composites as a function of system compliance Dental Materials 24(5) 645-652. 